\title{
The use of signature sequences in different proteins to determine the relative branching order of bacterial divisions: evidence that Fibrobacter diverged at a similar time to Chlamydia and the Cytophaga- Flavobacterium-Bacteroides division
}

\author{
Emma Griffiths and Radhey S. Gupta
}

Author for correspondence: Radhey S. Gupta. Tel: +1 9055259140 ext. 22639. Fax: +1905 5229033. e-mail:gupta@mcmaster.ca

Department of

Biochemistry, McMaster University, Hamilton,

Ontario, Canada L8N $3 Z 5$
The phylogenetic placement of the rumen bacterium Fibrobacter succinogenes was determined using a signature sequence approach that allows determination of the relative branching order of the major divisions among Bacteria [Gupta, R. S. (2000) FEMS Microbiol Rev 24, 367-402]. For this purpose, segments of the Hsp60 (groEL), Hsp70 (dnaK), CTP synthase and alanyl-tRNA synthetase genes, which are known to contain signature sequences that are useful for phylogenetic deterministic purposes, were cloned. Using degenerate oligonucleotide primers for highly conserved regions in these proteins, $1.4 \mathrm{~kb}$, $0.75 \mathrm{~kb}, 401 \mathrm{bp}$ and $171 \mathrm{bp}$ fragments of the Hsp70, Hsp60, CTP synthase and alanyl-tRNA synthetase genes respectively were amplified by PCR, and these fragments were cloned and sequenced. These primers, because of their high degree of conservation, could also be used for cloning these genes from other bacterial species. The Hsp70 homologues from different Gram-negative bacteria contain a 21-23 aa insert that is not found in any Gram-positive bacteria. The presence of this insert in the $F$. succinogenes Hsp70 supports its placement within the Gram-negative group of bacteria. A conserved insert in $F$. succinogenes Hsp60 that is commonly present in all bacterial species, except various Gram-positive bacteria, Deinococcus-Thermus groups and green nonsulphur bacteria, provides evidence that $F$. succinogenes does not belong to these taxa. A particularly useful signature consisting of a 4 aa insert is found in Ala-tRNA synthetase. This insert is present in all proteobacterial homologues as well as in homologues from species belonging to the Chlamydia and Cytophaga-Flavobacterium- Bacteroides (CFB) groups, but it is not found in homologues from any other groups of bacteria. The presence of this insert in $F$. succinogenes Ala-tRNA synthetase provides evidence that this species is related to these groups. However, two other signatures in CTP synthase and Hsp70 proteins, that are distinctive of the proteobacterial species, are not present in the $F$. succinogenes homologues. These results provide evidence that $F$. succinogenes does not belong to the proteobacterial division and thus should be placed in a similar position as the Chlamydia and CFB groups of species.

Keywords: bacterial evolution, phylogeny, phylogenetic placement, Fibrobacter succinogenes, fibrolytic bacteria 


\section{INTRODUCTION}

The rumen bacterium Fibrobacter succinogenes contains a number of cellulolytic and hemicellulolytic enzymes which are responsible for the digestion of the cell walls of forage plants in the bovine rumen (Cheng et al., 1991; Gokarn et al., 1997). Because of the established role of $F$. succinogenes as one of the major indigenous fibrolytic micro-organisms in ruminants, there is much interest in this species from the viewpoints of farm production efficiency, alternative waste management, and its evolutionary relationship to other bacteria (Asanuma et al., 1999; Cheng et al., 1991; Debroas \& Blanchart, 1993; Fields et al., 2000; Garcia-Vallve et al., 2000; Huang \& Forsberg, 1990; Shi et al., 1997; Wang et al., 2000; Weimer et al., 1999).

Based on its physiological characteristics, F. succinogenes, originally known as Bacteroides succinogenes, was previously considered to be a member of the Cytophaga-Flavobacterium-Bacteroides (CFB) division (Hungate, 1950). However, subsequent studies based on $16 \mathrm{~S}$ rRNA indicated that this and other related strains were not closely related to other species of Bacteroides, leading to their placement in a separate genus named Fibrobacter (Montgomery et al., 1988). A recent taxonomic classification based on $16 \mathrm{~S}$ rRNA places Fibrobacter in a separate division, distinct from the CFB group, originating from the common bacterial ancestor (Amann et al., 1992; Maidak et al., 1999; Ludwig \& Schleifer, 1999; Zinder, 1998). Our current understanding of the evolutionary relationships among prokaryotes is based almost entirely on 16S rRNA sequences (Hugenholtz et al., 1998; Maidak et al., 1999; Olsen \& Woese, 1993; Woese, 1987). Although, the phylogenetic trees based on $16 \mathrm{~S}$ rRNA have proven extremely useful in the placement of species into different groups, one of their main recognized limitations has been their inability to resolve the relationships among the major divisions within bacteria or to deduce how these groups branched from a common ancestor (Maidak et al., 1999; Ludwig \& Schleifer, 1999; Gupta, 1998a, 2000c). Recently, we have described a new approach based on the presence of shared conserved indels (or signature sequences) in different proteins for deducing the phylogenetic relationships among different bacterial groups, that complements this major deficiency of the rRNA trees (Gupta, 1998a, 2000a). By following the presence or absence of signature sequences in a number of different proteins in various divisions of bacteria, it is now possible to deduce logically how the major taxa within the Bacteria have branched from a common ancestor. The branching order for various divisions as determined from these studies is as follows: low-G + C Gram-positives $\rightarrow$ high$\mathrm{G}+\mathrm{C} \quad$ Gram-positives $\rightarrow$ Deinococcus - Thermus $\rightarrow$ green non-sulphur bacteria $\rightarrow$ cyanobacteria $\rightarrow$ spirochaetes $\rightarrow$ Chlamydia-CFB group $\rightarrow \delta, \varepsilon$-Proteobacteria $\rightarrow \alpha$-Proteobacteria $\rightarrow \beta$-Proteobacteria $\rightarrow \gamma$-Proteobacteria (Gupta, 1998a, 2000a, b).

The identified signature sequences in various proteins are also useful for deterministic purposes and based upon the presence or absence of these signatures, it is now possible to place with a high degree of confidence any given bacterial species within one of the main groups that could thus far be distinguished (Gupta, 1998a, 2000a, b). In the present study, we have used the signature sequence approach to understand the phylogenetic placement of $F$. succinogenes within the domain Bacteria. For this purpose, we have amplified by PCR and sequenced segments of the Hsp60 (groEL), Hsp70 $(d n a K)$, CTP synthase and alanyl-tRNA (Ala-tRNA) synthetase genes from $F$. succinogenes, which are known to contain useful signature sequences that are distinctive for different bacterial divisions (Gupta, 1998a, 2000a). The analyses of these signature sequences provide strong evidence that $F$. succinogenes shows a specific relationship to the Chlamydia and CFB groups of species. These signature sequences also provide evidence that this group of bacteria evolved after the branching of Grampositive bacteria, Deinococcus-Thermus groups, green non-sulphur bacteria, cyanobacteria and spirochaetes, but prior to the branching of Proteobacteria (Gupta, 1998a, 2000a).

\section{METHODS}

PCR amplification and sequencing. Purified DNA of $F$. succinogenes S85 (type strain; ATCC 19169) was generously made available to us by Dr C. Forsberg (Guelph University, Ontario, Canada) and Dr J. Yanke (Agriculture Canada Research Centre, Lethbridge, Alberta). Degenerate oligonucleotide primers, in opposite orientations, were designed for highly conserved regions in the indicated proteins that flanked the previously identified signature sequences. The regions of high sequence similarity suitable for primer design were identified by carrying out multiple sequence alignments of homologues for these proteins from different species. These PCR primers, because they correspond to highly conserved regions, can be used to amplify homologous gene fragments from different species. All primers were synthesized at MOBIX (The Central Facility of the Institute for Molecular Biology and Biotechnology, McMaster University).

Hsp70 gene. The PCR amplification of the Hsp70 gene fragment was carried out in two segments. Initially, a fragment of $1080 \mathrm{bp}$ was amplified using degenerate primers for two highly conserved regions in the Hsp70 protein (Galley et al., 1992). The forward primer was for the amino acid sequence GIDLGTT (nucleotide sequence 5'-GGNATHGAYYTNGGNACNAC- $3^{\prime}$ ) and the reverse primer for the sequence NPDEAVA (nucleotide sequence, 5'-GCNACNGCYTCRTCNGGRTT-3', where R represents A or G; Y represents C or $\mathrm{T}$; H represents $\mathrm{T}, \mathrm{A}$, or $\mathrm{C}$; and $\mathrm{N}$ represents $\mathrm{A}, \mathrm{G}, \mathrm{C}$, or $\mathrm{T}$ ). After this fragment was cloned and sequenced, the sequence was extended in the direction of the $3^{\prime}$-end by designing a specific forward primer near the $3^{\prime}$-end of the sequence $\left(5^{\prime}\right.$ AGGAACCGAACAAGACTG-3') and a degenerate primer in the reverse orientation for the conserved sequence QIEVTFD towards the C-terminal end of the protein (nucleotide sequence 5'-TCRAANGTNACYTCDATYTG-3') (Galley et al., 1992). This led to the amplification of a $361 \mathrm{bp}$ fragment whose sequence was combined with the original Hsp70 fragment to obtain a contiguous sequence of $1400 \mathrm{bp}$. The nucleotide and corresponding protein sequence were deposited in GenBank under the accession number AY017382.

Hsp60 gene. The amplification of the Hsp60 gene fragment 
was carried out using a degenerate forward primer for the amino acid sequence GPKGRN (5'-GGNCCNAARGGNA(C)GNAAYGT-3') and a degenerate reverse primer for the sequence AVKAPGFGE (5'-TCNCCRAANCCNGGNGCYTTNACNGC-3') (Rusanganwa et al., 1992). These primers amplified a $756 \mathrm{bp}$ fragment of the Hsp60 protein. Sequence data were deposited in GenBank under the accession number AY017380.

Alanyl-tRNA synthetase. A $171 \mathrm{bp}$ fragment of Ala-tRNA synthetase was amplified using a degenerate forward primer for the amino acid sequence FTNAGM (5'-TTYACNAAYGCNGGNATG-3') and a reverse primer for the sequence HTFFEM (5'-CATYTCRAARAANGTRTG-3'). Protein and nucleotide sequences were deposited in the GenBank database under the accession number AY017381.

CTP synthase. A $401 \mathrm{bp}$ fragment of CTP synthase was amplified using a degenerate forward primer for GICLGMQ $\left(5^{\prime}\right.$-GGNATHTGYYTNGGNATGCA-3') and a reverse primer for QFHPEF (5'-AAYTCNGGRTGRAAYTG-3'). Sequences for this gene and its corresponding protein were deposited in GenBank under the accession number AY017383.

PCR. PCR reactions, of $10 \mu \mathrm{l}$ volumes (approx. 0.2 $\mu \mathrm{g}$ DNA per reaction), were optimized for $\mathrm{Mg}^{2+}$ concentration (in the range of 1.5-4 mM) for each set of primers. PCR was carried out using a Techne Progene thermocycler, over 30 cycles $(15 \mathrm{~s}$ at $94{ }^{\circ} \mathrm{C}, 15 \mathrm{~s}$ at $55^{\circ} \mathrm{C}, 1 \mathrm{~min}$ at $72^{\circ} \mathrm{C}$ ) with an initial $1 \mathrm{~min}$ hot start at $94^{\circ} \mathrm{C}$, and a final extension step $\left(15 \mathrm{~s}\right.$ at $94^{\circ} \mathrm{C}, 15 \mathrm{~s}$ at $55^{\circ} \mathrm{C}, 7 \mathrm{~min}$ at $\left.72{ }^{\circ} \mathrm{C}\right)$. The DNA fragments of the expected size were purified from $0 \cdot 8 \%(\mathrm{w} / \mathrm{v})$ agarose gels (using a GENECLEAN kit), and subcloned into the plasmid pCR TOPO 2.1 using a TA cloning kit (Invitrogen). After transformation of Escherichia coli JM109 cells with the plasmids, the inserts from a number of positive clones were sequenced. The fragments of less than $0.6 \mathrm{~kb}$ in length were sequenced by the dideoxy chain termination method using a T7 sequencing kit (Pharmacia). The larger fragments were sequenced by MOBIX. Sequences of all cloned fragments were run through a BLAST search to ensure the DNA was from a novel source.

Signature sequence analysis. The nucleotide sequences for the four F. succinogenes genes were translated and added to multiple alignments of homologous proteins (sequences from various known bacteria acquired from BLAST searches, www.ncbi.nlm.nih.gov). Alignments were carried out with either ALIGN or CLUSTAL w software.

\section{RESULTS}

\section{Signature sequences and their usefulness in deducing evolutionary relationships}

Signature sequences in proteins, defined as conserved inserts or deletions (i.e. indels) that are restricted to specific taxa provide a powerful means to deduce the evolutionary relationship among distantly related organisms (Gupta, 1998a, b). In phylogenetic trees based on most gene sequences, including that of the $16 \mathrm{~S}$ rRNA, evolutionary relationships between the major taxa within each domain are generally not resolved; this has been recognized as a major weakness or limitation of the tree construction approach (Olsen \& Woese, 1993; Ludwig \& Schleifer, 1999; Gupta, 2000c). The inability of the phylogenetic trees to resolve such relationships is in large part due to the dependence of the derived inference upon a number of different variables and assumptions (e.g. reliability of the sequence alignment, regions of sequences that are retained or excluded in phylogenetic analysis, number and range of species included, differences in the evolutionary rates, base compositional differences, phylogenetic methods employed, etc.) (Gupta, 1998b). Hence, there is a clear need for alternative approaches that are minimally affected by the above variables and assumptions, and which are capable of resolving such relationships. The presence of shared signature sequences in proteins provides such an approach.

The use of the signature sequence approach for understanding the evolutionary relationship is based on the rationale that when a conserved indel of defined length and sequence, and flanked by conserved regions to ensure that the observed changes are not due to incorrect alignment or sequencing errors, is found at the same position in homologues from different species, then the simplest and most parsimonious explanation for this observation is that the indel was introduced only once during the course of evolution and then passed on to all descendants. A well-defined indel in a gene or protein thus provides a very useful milestone for evolutionary events, since all species emerging from the ancestral cell in which the indel was first introduced are expected to contain the indel, whereas all species that existed before this event or which did not evolve from this ancestor will lack the indel (Gupta, 1998a, c). To interpret whether a given indel in a particular protein is the result of an insertion or deletion event, and its possible implications regarding the relative branching order of different groups, a reference point is essential. Such a reference point is provided by the root of the prokaryotic tree. Several lines of evidence show that the root of the prokaryotic tree lies between the Archaea and Grampositive bacteria (Iwabe et al., 1989; Brown \& Doolittle, 1995; Gupta, 1998a, b, 2000b), both of which are prokaryotic organisms bounded by a single membrane. Based on this rooting, the signature sequences in various proteins can be clearly interpreted and the branching order of different groups from the common ancestor can be logically deduced as described in earlier work (Gupta, 1998a, 2000a).

\section{Deducing the phylogenetic placement of F. succinogenes}

To employ the signature sequence approach for determining the phylogenetic placement of any given species, one begins by successively examining the signature sequences in different proteins that exclude the placement of the species in certain groups and at the same time show specific affinity of the species, or narrow down its placement, in certain other group(s) (Gupta, 1998a). One prominent signature consists of an indel of 21-23 aa, present in the N-terminal quadrant of Hsp70 protein, which distinguishes all true Gram-negative bacteria (defined by the presence of an inner and an outer membrane) from Gram-positive bacteria (Gupta, 1998a, 2000a). In earlier work, we have presented evidence that this indel represents an insert in the Gram- 


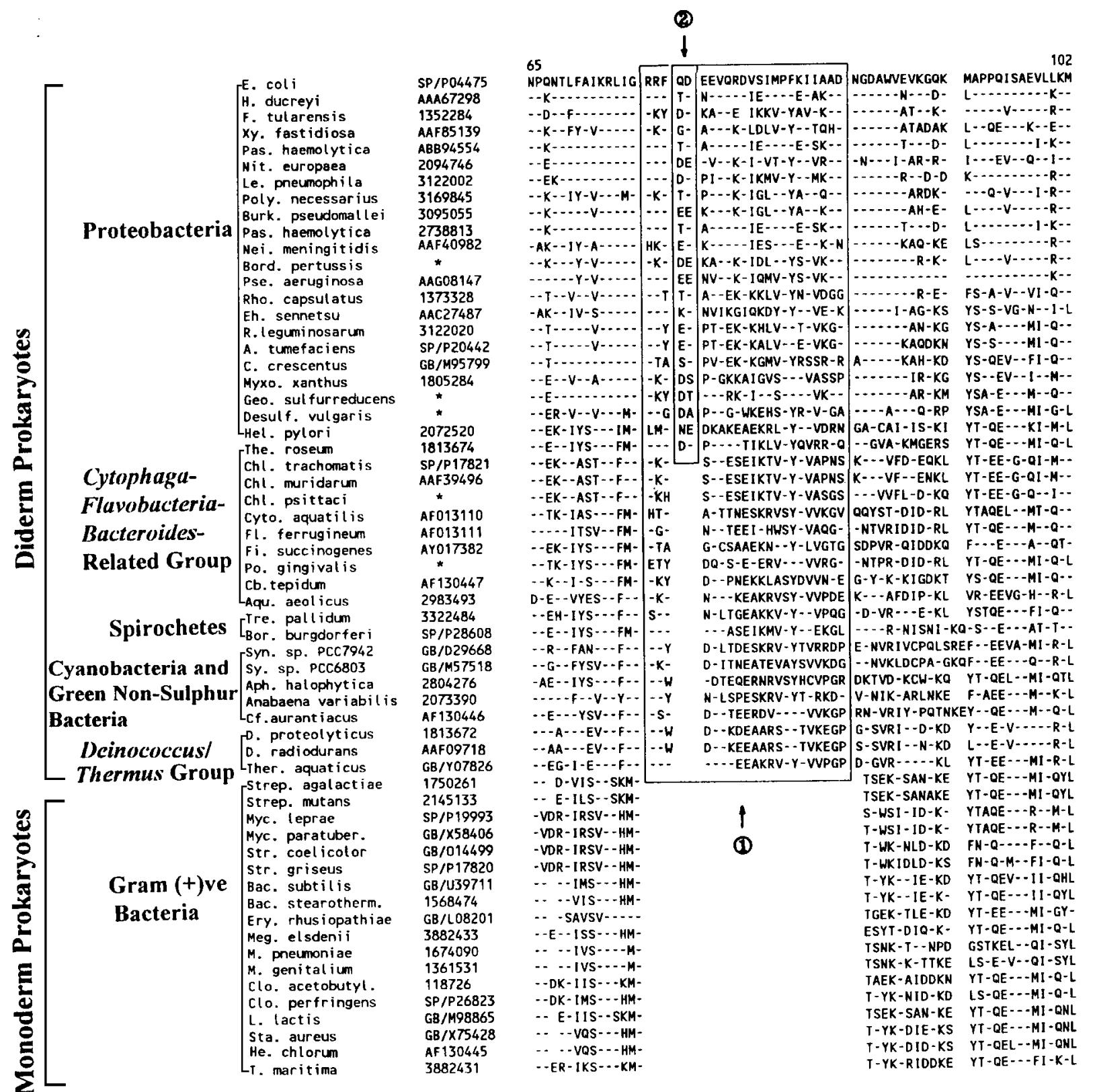

Fig. 1. Partial alignment of Hsp70 sequences showing a signature sequence consisting of an insert of 21-23 aa (box 1), which is specific for Gram-negative (or diderm) bacteria. The presence of this insert in $F$. succinogenes provides evidence that it is a Gram-negative bacterium. A smaller 2 aa insert (box 2) within the large insert is distinctive of the proteobacterial species. This insert is absent in $F$. succinogenes. Dashes indicate an identical amino acid to that found in the $E$. coli sequence shown on the top line. Accession numbers of the sequences are shown in the second column. The asterisks (*) denote sequences identified by BLAST search from the NCBI Unfinished microbial genome database (www.ncbi.nlm.nih.gov/BLAST/unfinishedgenome/html). Abbreviations used are as follows: A., Agrobacterium; Aph., Aphanothece; Aqu., Aquifex; Bac., Bacillus; Bor., Borrelia; Bord., Bordetella; Burk., Burkholderia; C., Caulobacter; Cb., Chlorobium; Chl., Chlamydia; Cf., Chloroflexus; Clo., Clostridium; Cyto., Cytophaga; D., Deinococcus; Desulf., Desulfovibrio; E., Escherichia; Eh., Ehrlichia; Ery., Erysipelothrix; F., Francisella; Fi., Fibrobacter; Fl., Flavobacterium; Geo., Geobacter; H., Haemophilus; He., Heliobacterium; Hel., Helicobacter; L., Lactococcus; Le., Legionella; M., Mycoplasma; Meg., Megasphaera; Myc., Mycobacterium; Myxo., Myxococcus; Nei., Neisseria; Nit., Nitrosomonas; Pas., Pasteurella; Po., Porphyromonas; Poly., Polynucleobacter; Pse., Pseudomonas; R., Rhizobium; Rho., Rhodobacter; Sta., Staphylococcus; Str., Streptomyces; Strep., Streptococcus; Sy., Synechocystis; Syn., Synechococcus; T., Thermotoga; The., Thermomicrobium; Ther., Thermus; Tre., Treponema; Xy., Xyllela.

negative bacteria rather than a deletion in the Grampositive bacteria (Gupta \& Singh, 1992; Gupta \& Golding, 1993; Gupta, 1998a), indicating the ancestral nature of the Gram-positive group. Hsp70 is a highly conserved protein, which carries out an essential chaperone function in the cell (Lindquist \& Craig, 1988). 


\section{Proteobacteria}

\section{Cytophaga- Flavobacteria- Bacteroides- Related Group}

\author{
Spirochetes \\ Cyanobacteria
}

\section{Deinococcus/ Thermus Group}

\author{
Gram (+)ve \\ Bacteria
}

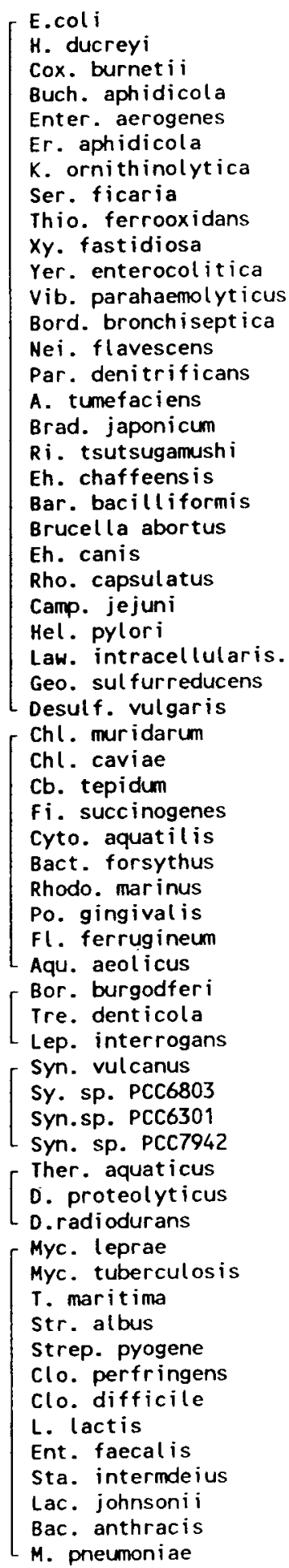

\begin{tabular}{|c|c|c|}
\hline & 144 & 778 \\
\hline 6139 & $\begin{array}{l}\text { IAQVGT ISA } \\
- \text { E....... }\end{array}$ & \begin{tabular}{|c|c} 
N & SDETVGKL I AEAMDKVGKEGVI IVE \\
- & -
\end{tabular} \\
\hline $\begin{array}{l}1294 \\
9421\end{array}$ & -E & - -KSI-DI - \\
\hline 9177 & $T \ldots \ldots$ & $-A--K--A \cdots--E--N D \cdots$ \\
\hline 026143 & $\ldots \ldots$ & $5 \cos 20$ \\
\hline 37 & 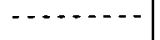 & 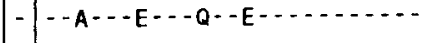 \\
\hline 1 & ........ & - \\
\hline 21 & $\cdots$ & - \\
\hline 34 & $v-\ldots . .$. & $--D S I--I \cdots--E--N-\cdots$ \\
\hline 849 & $\cdots A-\cdots$ & $\cdots-S I-N I-\cdots-K \cdots-$ \\
\hline 71 & ........... & $-\mid--S--E-Q Q-E-\cdots$ \\
\hline 28 & $\ldots \ldots$ & $-\mid--$ AS - -NI $\cdots-$-ER - RD - - - \\
\hline & $\cdots-\operatorname{s-n}$ & $-(-A S I-Q I-D-\ldots \ldots$ \\
\hline CAAB & 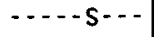 & $\cdots-Q--A I \cdots-E \cdots$ \\
\hline & 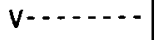 & - GESF I -QQ - . - Q QR - - N - $\cdots$ \\
\hline & $v-\ldots \ldots$. & - GERQI - LD - - - QR - -N- \\
\hline & $\ldots \ldots$ & $-\quad G-A E I--F-S D \cdots K-\cdots N-\cdots$ \\
\hline P16 & $\cdots-A-V-S$ & - G-RE I-EK--N- KQ- -Q - \\
\hline & $\cdots-\cdots$ & G-KN I - SK- -QCVQE - - D- \\
\hline & $\cdots-A-\cdots$ & $G A-D I=-M--D--E-C-N \cdots$ \\
\hline & v $\ldots . .$. & AEAEI $-M-\cdots$ \\
\hline & $\cdots-A-\cdots$ & - G-KNI -VK- QCVQE- - -D- - . \\
\hline & V........ & - GEAQI -RF - -D -SQ - - N - . \\
\hline & $\cdots-\cdots$ & $-\mid--K I-N--D--E---D-\cdots$ \\
\hline & $-T--A-\cdots$ & {$[\cdots D-E-D D \cdots$} \\
\hline & .......... & 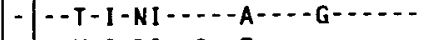 \\
\hline & $\cdots-$ & $D I--Q--E-\cdots-D$ \\
\hline ネ & $\ldots \ldots$ & $-2-2+1-4 I-\ldots-2, \ldots$ \\
\hline 43 & $\cdots-A-\cdots$ & $-N-A E I-N-\cdots-E-\cdots-S-\cdots$ \\
\hline & $\cdots-A-$ & $N-A E I-N-C D-E=-N-S-$ \\
\hline & -........ & -EWCDCD \\
\hline & $\cdots-A-\cdots$ & $-N-P E I-E-L-N--E-\cdots-N D \cdots--I-$ \\
\hline & $-K-I /$ & $-E-D-T-F A-\cdots--D$ \\
\hline & $-E H-A$ & 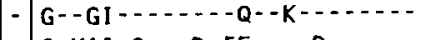 \\
\hline & $\cdots$ - $-\cdots$ & $-G-K A I-Q-\cdots D-F E-\cdots-$ \\
\hline & -EH-AK-.- & $-G--N I-S-\cdots-R-K \cdots$ \\
\hline & $-Q--A-\cdots$ & $\cdots-F A-\cdots$ \\
\hline & $-E--A$ & $--I--D--E E--D-\cdots$ \\
\hline P275 & $\cdots$ AS & 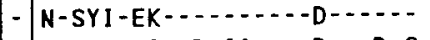 \\
\hline & $V-H-A S V--$ & $-N-A E I--I-D-I A-\cdots-D--D-G$ \\
\hline$L 14682+2$ & $--N-A S-\cdot-$ & $N-N-I-N---D-C-1-D-$ \\
\hline & $\cdots A A \cdots$ & G $N--E--R M--D-\cdots$ \\
\hline & $\cdots--1$ & G $N--E--Q M--N-\cdots-Q-\cdots-S L-$ \\
\hline & 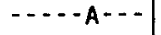 & G $\quad N-F E--Q M--D-\cdots-K-C S L-$ \\
\hline & - . - A- - - & G $N-F E--Q M--D-\cdots$ \\
\hline & $-E E-A-\cdots$ & \rfloor$_{N-P E}-\cdots-D-E-\cdots-I \cdots$ \\
\hline & $-K K-A G=-$ & $N-P Q--E E--S-\ldots \ldots$ \\
\hline & $-K K-A$ & QQE- - S - - - \\
\hline & $\because$-ATA & - n \\
\hline & $--A T$ & 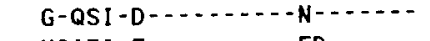 \\
\hline & $--\mathrm{H}-\mathrm{A}$ & $E-\cdots-C-C D$ \\
\hline & $--A-A A L--$ & $Q-K Q-E \cdots$ \\
\hline 584 & $\cdots$ - AAV-S & RS $-K--E Y-S-\cdots-E R=-N D---I-$ \\
\hline & $\because R-A A \cdots$ & $A--K I+\cdots-D--E--N \cdots$ \\
\hline & $-S-A S-\cdots$ & $G--E-\cdots-E I-D D \cdots$ \\
\hline$x 71132$ & $\cdots-V-V-S$ & RS-K--EY-SD--ER--SD- - I I- \\
\hline & & 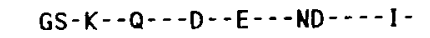 \\
\hline & $-\cdots-A-$ & $A--E--R Y-S-\cdots E---N D-\cdots S I-$ \\
\hline AAF & & ASTE - - N- -RD - -E - - HD - - \\
\hline & 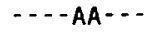 & $A--E-Q-\cdots-E R--N D \cdots$ \\
\hline P78012 & $-E--A A--S$ & GSKEI $\cdots-$ Q - AL - - N - - TD \\
\hline
\end{tabular}

Fig. 2. Excerpts from the Hsp60 sequence alignment showing a signature sequence that distinguishes Gram-positive bacteria, Deinococcus-Thermus and green non-sulphur bacteria from the other groups of bacteria. The presence of this insert in $F$. succinogenes provides evidence that it belongs to the latter group. Dashes denote identity to the amino acid in the top line. Additional abbreviations in the species names are as follows: Bact., Bacteroides; Bar., Bartonella; Brad., Bradyrhizobium; Buch., Buchnera; Camp., Campylobacter; Cox., Coxiella; Ent., Enterococcus; Enter., Enterobacter; Er., Erwinia; K., Klebsiella; Lac., Lactobacillus; Law., Lawsonia; Lep., Leptospira; Par., Paracoccus; Rhodo., Rhodothermus; Ri., Rickettsia; Ser., Serratia; Thio., Thiobacillus; Vib., Vibrio; Yer., Yersinia. 


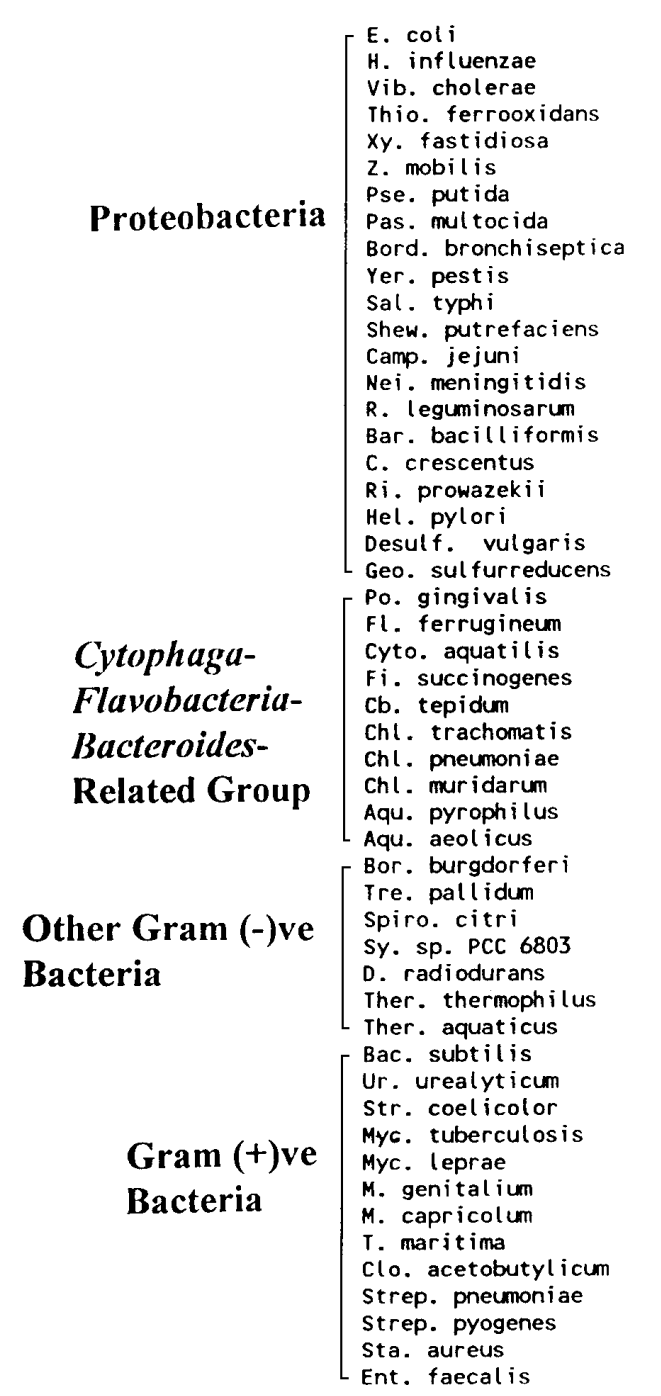

\begin{tabular}{|c|c|c|c|}
\hline & 57 & & 100 \\
\hline 145220 & RNYSRATTSQRCVRA & GGKH & NDLENVGYTARHHTFFEMLGNFSFG \\
\hline 1174496 & $-P-\ldots-A-\cdots-P$ & $\cdots$ & $\mid \begin{array}{lll}-1 & -1\end{array}$ \\
\hline 2500962 & $-A-T---A-\cdots \cdot$ & $-\cdots$ & $-\cdots-F-$ \\
\hline 1263913 & -P-R--VS-- - -M- - & & (- n \\
\hline AAF8 & -SEV-VADV-C-L - & -... & -- DS $\ldots . .$. \\
\hline AAD53924 & $-P-K T--S--K-\cdots$ & $\cdots$ & 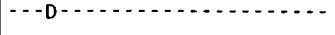 \\
\hline ^ & $-A-T--V-\cdots K \cdots$ & & $\ldots \ldots$ \\
\hline * & $-P \ldots-D A \cdots$ & - & $-\ldots . . .$. \\
\hline ^ & $A-K \cdots---S$ & 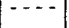 & D - - . - n \\
\hline * & A & 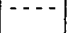 & $\cdots$ \\
\hline * & - & $\cdots$ & 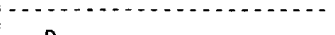 \\
\hline * & -S-T-D-A- - - & $-\cdots$ & 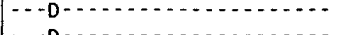 \\
\hline AB75143 & P-PP-K-SC-T-I - & $-\cdots$ & $\cdots$ D $\cdots \cdots$ \\
\hline 5015 & $-P \cdots---A-K-\cdots$ & $\cdots$ & $M$ \\
\hline 135091 & $-S--T-\cdots--K--V$ & $-\cdots$ & 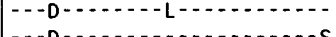 \\
\hline 3622 & HS-N- - - A-K- - - & $-\cdots$ & BD \\
\hline & HP--AS--K-.-- & $\cdots$ & 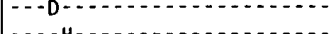 \\
\hline e1343125 & $-S-N K-V=--K S L=-$ & $\cdots$ & 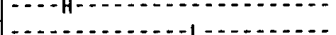 \\
\hline 23144 & PSIP-CAS- - L-M-- & $\cdots$ & 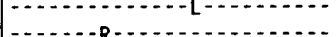 \\
\hline & $K-A-\cdots-K-L$ & $\cdots$ & 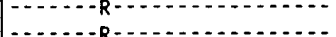 \\
\hline * & K-D-V--C- -K- - - & con & 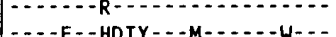 \\
\hline ״ & $A K-T-V A D--K-L-V$ & s-.. & $-2-{ }^{-2}$ \\
\hline AF 130451 & ASA-VADT - $K-L-V$ & s-a & 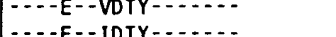 \\
\hline AF130452 & PKSP-IADT - K-L-V & S-.- & $-A$ - \\
\hline$\underset{\star}{\text { AY017381 }}$ & $\begin{array}{l}K V G=-C N--K-L-V \\
-E-T \cdots A D T-K-I--\end{array}$ & $\begin{array}{ll}S--- \\
\text { S-.. }\end{array}$ & 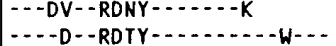 \\
\hline 6758113 & TS-T-...... K- $\cdots$ & $\ldots$ & $\ldots .-H-S+-[\ldots \ldots$ \\
\hline AAD 19030 & $5-\cdots---k-1--$ & $\cdots$ & $--D--H-S-L-\ldots$ \\
\hline AAF39003 & TS-T-- - - K-1-- & $\cdots$ & 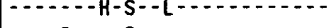 \\
\hline 4587716 & $-P-K-\cdot-S C-K-L-V$ & s-.. & 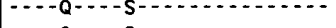 \\
\hline 2983727 & $-P-K-\cdot-S C-K-L-V$ & S--- & 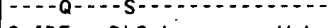 \\
\hline 2688110 & PSGDMLVNV-K-L-T & & G-IDE-- DLS-L- - - - - W-L \\
\hline AE001269 & PAGT - LVNA $-K-L-T$ & & G-IDA--DNS -L- \\
\hline AAC45550 & TPPP-L N--KSI-T & & 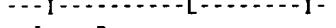 \\
\hline 1653611 & AEFP- - - K- $-1-T$ & & 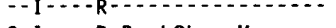 \\
\hline AAF 11848 & QPSK-V--A-K---V & & G-I - - - R-R- LSL - - M- \\
\hline 2500960 & $-E W R-V--C-E-L-V$ & & G-I - - - - R-S- $-N-Y-\ldots$ \\
\hline 1565288 & -EWR-V--C-E-L-V & & $G-I-\cdots-R-S--N-Y \cdots \cdots$ \\
\hline 2635186 & PENP - I VNA - KAI - T & & 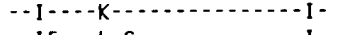 \\
\hline AAF30778 & PPHK - LVN- -KAI - T & & 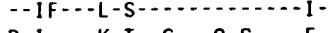 \\
\hline 3381 & PPFD-ATSV-K- - - T & & $P-1---K-T-G--G-C-\cdots-F-$ \\
\hline & $P P-P T--S I-K-I-T$ & & $P-I D E--I-T--N--Q-A-\cdots$ \\
\hline 31360 & $P P-A T=-S I-K-I-T$ & & $P-I D D--I-T--N---Q-A \cdots \cdots$ \\
\hline 145 & PPSK-LVNA-I-LrV & & $\cdots I--F-S--Q-L-\cdots \cdots \cdot-1-$ \\
\hline 10 & PPSP-L-N- -KAI - T & & 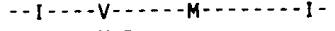 \\
\hline 81959 & $P V-T-V A-C-K-L-T$ & & V-I $-\cdots-K-P \ldots \ldots$ \\
\hline * & PPKT $-V--C-K-I-T$ & & G-I - I-K-S-G- - - - . \\
\hline * & PENP-I -NA-KAI - T & & $\cdots 1-\cdots-K-\cdots-M-\cdots,--1-$ \\
\hline « & PENP - I -NA-KS I - T & & $--1 \cdots-K-\cdots--M-\cdots,-$ \\
\hline & -IVN- -KAI-T & & 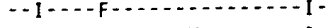 \\
\hline * & PENP-I -NA-KSI - T & & $\cdots I-\cdots K-\cdots-M-\cdots+-1-$ \\
\hline
\end{tabular}

Fig. 3. Signature sequence in the alanyl-tRNA synthetase gene, which is present only in the Proteobacteria and the Chlamydia and CFB groups of species. The presence of this insert in $F$. succinogenes indicates a relationship to these taxa. Dashed lines indicate an identical amino acid match to that of $E$. coli, given on the top line. Additional abbreviations are: Sal., Salmonella; Shew., Shewanella; Spiro., Spiroplasma; Ur., Ureaplasma; Z., Zymomonas.

Homologues of Hsp70 are present in all bacterial genomes sequenced to date, including those from the mycoplasmas, which contain a minimal gene complement (Fraser et al., 1995). To examine the presence of this signature sequence in $F$. succinogenes, a $1.4 \mathrm{~kb}$ fragment of the Hsp70 gene was amplified by PCR. Sequencing of this fragment revealed that F. succinogenes Hsp70 contained the 21 aa insert (Fig. 1), thus providing evidence that it belonged to the Gramnegative group of prokaryotes. The placement of $F$. succinogenes within the Gram-negative bacteria based on this signature is also in accordance with the observations that this species shows a negative Gram-staining reaction and possesses an outer membrane characteristic of Gram-negative bacteria (Holdeman et al., 1984).
Within Gram-negative bacteria two of the deepest branching divisions, Deinococcus-Thermus and green non-sulphur bacteria, can be distinguished from the others based on a signature present in the Hsp60 (GroEL) protein (Gupta, 1998a). This signature consists of a 1 aa insert that is commonly shared by species from various other groups of Gram-negative bacteria, including different groups of Proteobacteria, the Chlamydia and CFB groups, spirochaetes and cyanobacteria, but is not present in green non-sulphur bacteria and Deinococcus-Thermus (Gupta, 1998a). This insert, as expected, is also not found in any Gram-positive bacteria. Similar to Hsp70 protein, the Hsp60 protein is also a highly conserved protein found in all bacteria (Gupta, 1995), and the indicated signature is highly 


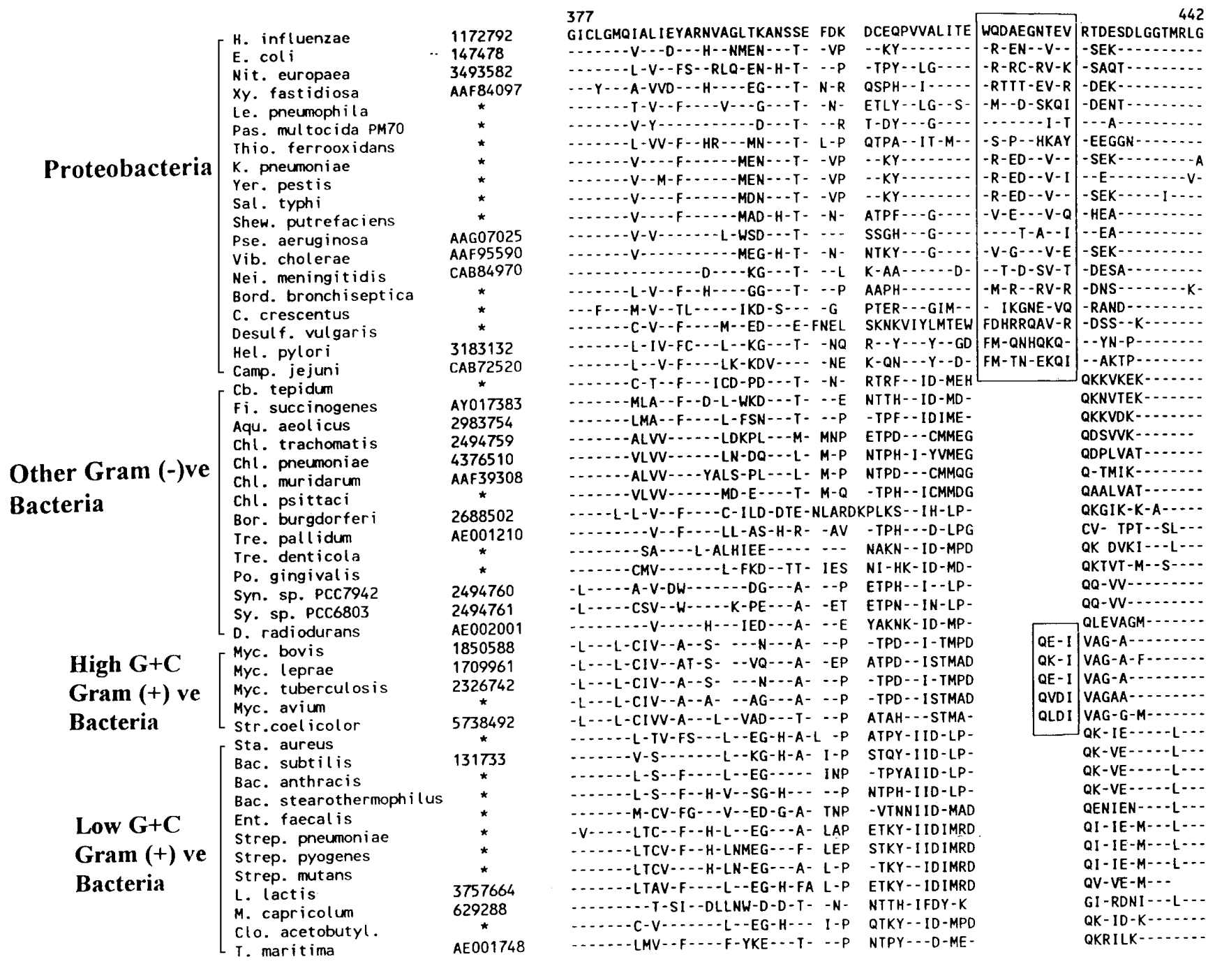

Fig. 4. A signature sequence consisting of a 10 aa insert in CTP synthase which is distinctive of the proteobacterial species. The absence of this insert in $F$. succinogenes provides evidence that it does not belong to the proteobacterial group.

reliable, with no exceptions observed in more than 200 sequences available from different bacterial species (Gupta, 2000a; unpublished results). To examine the presence of this signature in F. succinogenes, a $756 \mathrm{bp}$ fragment of the Hsp60 gene was amplified by PCR using degenerate primers based on highly conserved regions in the Hsp60 protein. The sequence alignment of a portion of this region from $F$. succinogenes with other bacterial species is presented in Fig. 2. As seen, the Hsp60 from F. succinogenes contains the indicated signature, providing evidence that this species does not belong to the green non-sulphur bacteria or the Deinococcus-Thermus groups.

We next examined the presence in F. succinogenes of a signature sequence found in the Ala-tRNA synthetase gene. The signature in this protein consists of a 4 aa insert that is uniquely found only in the homologues from various Proteobacteria and the Chlamydia and CFB groups of species (Gupta, 2000a). Ala-tRNA synthetase, which carries out an essential role in protein synthesis, is also found in all completed prokaryotic genomes (Gupta, 2000a). Using degenerate primers for highly conserved regions in this protein which flank this sequence signature, we successfully amplified a $171 \mathrm{bp}$ fragment that corresponded to the Ala-tRNA synthetase gene. The alignment of the translated sequence with other species shows that this insert is present in the AlatRNA synthetase from F. succinogenes (Fig. 3). In view of the specificity of this sequence signature, the presence of this insert in F. succinogenes provides strong evidence that this species belongs to one of these two groups of bacteria.

A number of signatures have been identified in proteins that are specific for the proteobacterial division and serve to distinguish members of this group from other divisions of bacteria. The protein CTP synthase contains a $10 \mathrm{aa}$ insert that is uniquely shared by all proteobacterial species, but is not found in the Chlamydia and 


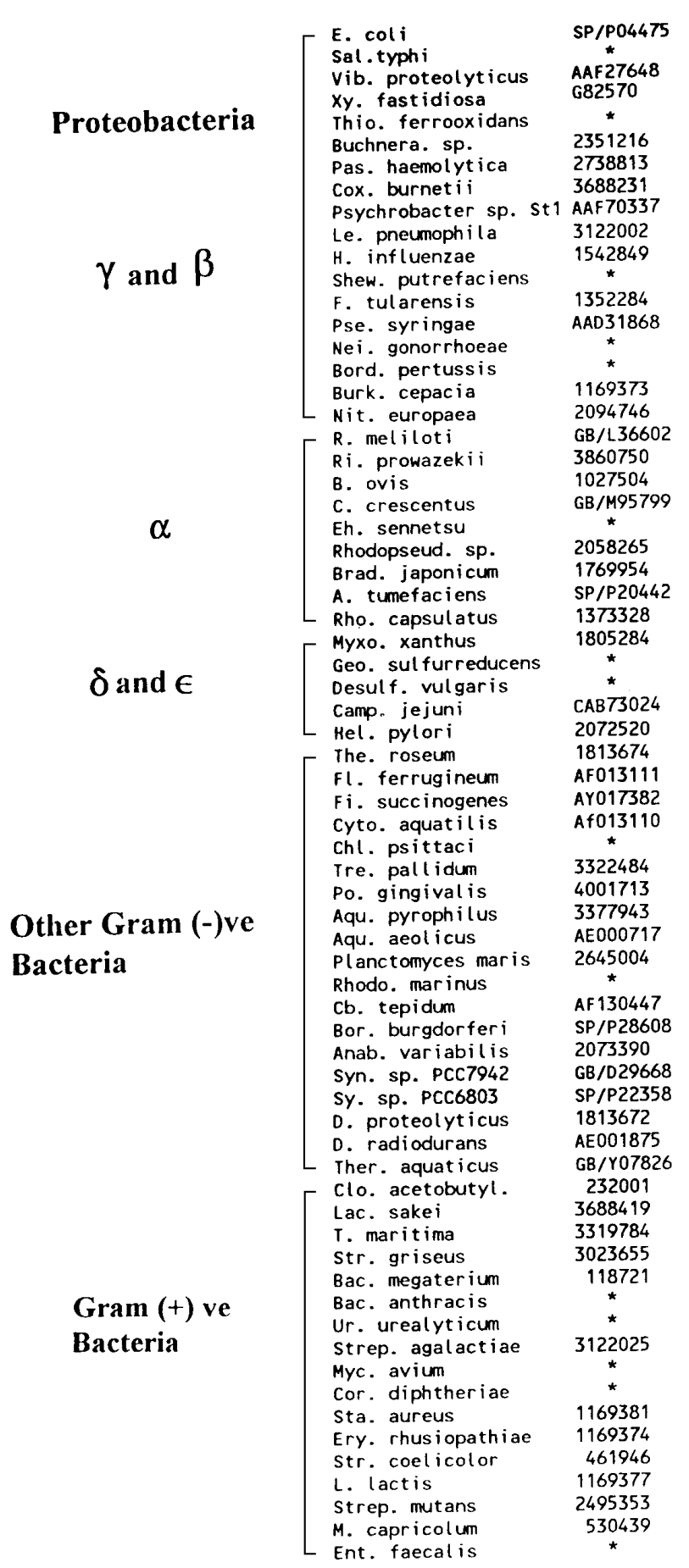

\begin{tabular}{|c|c|c|}
\hline & & \\
\hline I IEI & DEVD & GEKTFEVLATNGDTHLGGEDFD \\
\hline & & \\
\hline & A - . - & $\ldots-\ldots \ldots$ \\
\hline & A-ME & - HO-...S. \\
\hline & & $k-\ldots$. \\
\hline & $\mid \begin{array}{l}-\mathrm{NF}- \\
\mathrm{A}-\mathrm{.}\end{array}$ & 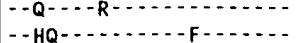 \\
\hline$v-$ & $A D-$. & 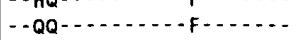 \\
\hline & A - . & $-H Q-C H C-F$ \\
\hline & $-\mathrm{NF}-$ & $--Q \ldots \ldots, G-N-$ \\
\hline & $-\mathrm{SN}-$ & 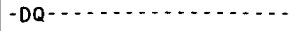 \\
\hline & SD - - & -DNQI -. -S -....-F- \\
\hline$-v-v--$ & & $-H Q \cdots \ldots . . . .-F-$ \\
\hline & ANL - & 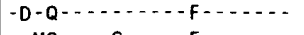 \\
\hline & $A D--$ & 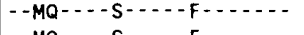 \\
\hline & $A D \cdots$ & - MQ-N.-S-....F- \\
\hline & & - $\mathrm{HQ}$-........F- \\
\hline & & 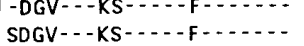 \\
\hline & & -DGV - - KS - . - F - - \\
\hline$-V--L=-$ & & -DGV-.-KS-...FF- \\
\hline & & $-D G Q---K \cdots \ldots$ \\
\hline$v$ & & - KS $\cdots--F$ \\
\hline$-L--$ & & $-D G V-\cdots K S-\cdots-F--$ \\
\hline & & $--K S \cdots-F_{-1}$ \\
\hline$\cdots \mathrm{VL}--$ & & DDGL $\cdots-$ KS $\cdots-\cdots$ \\
\hline & & NAGV - -KS $\cdots$ \\
\hline$\cdots-L-L$ & & -KS $-\cdots-F$ \\
\hline$\cdots L-V$ & & -DNVV- -R - \\
\hline & & $\cdots-$ G-NAF- - -D - . \\
\hline VL-T & & -DNWV-D-G-AF-D-D - \\
\hline & & S-GV-Q- - - - - Y-TPLVH- - \\
\hline & & - KS- \\
\hline 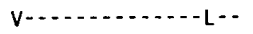 & & -DGM-SAK -..-- - M- - -DN - - \\
\hline$V L-L$ & & 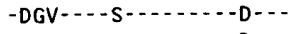 \\
\hline$--L$ & & 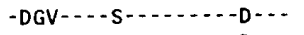 \\
\hline & & KS- $-\cdots$ \\
\hline$-L-L$ & & $\cdots$ Ks - - n \\
\hline$-L-G$ & & $-K-A-\ldots \ldots$-ANI - \\
\hline$-\mathrm{LE}$ & & KV-A- - - ANI - \\
\hline LDV & & $--S-S-\cdots+D-W-$ \\
\hline & & - . - DN- - \\
\hline$--L-L$ & & $--K S-D-\ldots . .-D-1$ \\
\hline & & 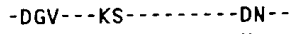 \\
\hline & & $-2--0 \cdots-n-1$ \\
\hline & & $-K--S-\cdots Q--N-$ \\
\hline & & SW.....-D \\
\hline & & $S--S--A-\cdots$ \\
\hline & & $A-\cdots, A-C$ \\
\hline & & $--K-S-\cdots-S-M-$ \\
\hline & & $S--n-\cdots$ \\
\hline & & $1-S-C-R=-D \cdots$ \\
\hline & & $I-A-N N \cdots D-\cdots$ \\
\hline & & $K-\cdots-N-\cdots-W-$ \\
\hline$-L$ & & $-R--A--N R-\cdots D-\cdots$ \\
\hline & & $A--N R=-D \cdots$ \\
\hline & & $\cdots-2-2-n-\cdots$ \\
\hline & & D-C-A-NK-C-D - \\
\hline$\cdots$ & & $-R=-2--N-C-D-h-$ \\
\hline & & $-R--A--N E--D-W-$ \\
\hline & & $-n K=-2 m-1$ \\
\hline & & $\cdots-S-A--N K \cdots-D$ \\
\hline LL.- & & $-D G V W--K-\cdots-N N---D-W-$ \\
\hline$--V--L-L$ & & $-D G V-D \cdots-A-N N K-\cdots-D--$ \\
\hline$-V--L-L$ & & $-D G V-D \cdots A--N K--D N--$ \\
\hline & & - I - S-NNK-C-DN-- \\
\hline & & \\
\hline
\end{tabular}

Fig. 5. A 4 aa insert in Hsp70 that is specific for the $\beta$ and $\gamma$-subdivisions of Proteobacteria. This insert is also absent in $F$. succinogenes, supporting the inference from CTP synthase and Hsp70 signatures that this species is not a part of the proteobacterial group. Additional abbreviations are: Anab., Anabaena; B., Brucella; Cor., Corynebacterium, Rhodopseud., Rhodopseudomonas.

CFB groups, or other divisions of bacteria (Gupta, 2000a). Using degenerate primers for highly conserved regions in CTP synthase, we were able to amplify a $401 \mathrm{bp}$ fragment for the CTP synthase gene from $F$. succinogenes which contained this sequence region. The alignment of the translated sequence from F. succinogenes with other bacterial species is presented in Fig. 4. From the alignment it is clear that the 10 aa insert common to proteobacterial species is not present in Fibrobacter. In addition to CTP synthase, Hsp70 also 
contains a 2 aa insert within the large insert that is distinctive of the proteobacterial species (box 2 in Fig. $1)$. This 2 aa insert is not found in the F. succinogenes Hsp70 either, providing further evidence that Fibrobacter does not belong to the proteobacterial group. The Hsp70 protein contains yet another signature consisting of a 4 aa insert that is specific only for the $\beta$ and $\gamma$ subdivisions of Proteobacteria (Fig. 5) (Gupta, 2000a). As expected, the Hsp70 homologue from F. succinogenes does not contain this insert, providing additional evidence that Fibrobacter does not belong to these groups of Proteobacteria. Based on the signature sequences in different proteins, it is thus clear that Fibrobacter is specifically related to the Chlamydia and CFB groups of species.

\section{DISCUSSION}

In the past 15-20 years, phylogenetic analyses based on $16 \mathrm{~S}$ rRNA sequences have become the primary means for understanding the evolutionary relationships among prokaryotes. Based on the branching positions of species in the rRNA trees, a number of major groups or divisions among bacteria have been identified (Woese, 1987 ; Olsen \& Woese, 1993; Ludwig \& Schleifer, 1999). The analysis based on 16S rRNA sequences has proven invaluable and generally quite successful in the assignment of various bacterial species into one of these groups (Maidak et al., 1999). However, one major limitation of the phylogenetic trees based on 16S rRNA (or those based on different protein sequences) is that they are unable to resolve reliably the branching orders of higher taxa or main groups within bacteria (Gupta, 1998a; Ludwig \& Schleifer, 1999; Olsen \& Woese, 1993). Due to this limitation, a number of important issues that are central to understanding bacterial phylogeny still remain unresolved. Such questions include: (i) how the major divisions within the Bacteria are related to each other, and (ii) in what order these groups branched off from the common ancestor (Gupta, 2000b, c). The inability of the rRNA trees (or those of various proteins) to resolve these relationships has led to growing acceptance of the notion that these problems are basically unsolvable and that all of the main groups within the Bacteria probably branched off directly from the common ancestor (Doolittle, 1999; Ludwig \& Schleifer, 1999; Woese, 1998).

In this context, the signature sequence approach developed in our recent work provides a new and powerful method for examining the evolutionary relationships among the species (Gupta, 1998a, 2000a). In contrast to the phylogenetic trees, where the derived inference is based upon a large number of variables (e.g. reliability of the alignment, regions of sequences that are retained or excluded, number and range of species included, differences in evolutionary rates, base compositional differences, phylogenetic methods employed, etc.) which contributes to the uncertainties of the end results (Gupta, 1998a), the evolutionary inference in this new approach is based entirely on the presence or absence of highly conserved sequence features. These sequence characteristics comprise indels of defined lengths present in highly conserved parts of the molecules, which makes interpretation of the data generally quite simple, unambiguous and based on minimal assumptions (Gupta, 1998a). Based upon a large number of conserved indels in various proteins that have been introduced at various stages in evolution, it is now possible to clearly define most of the major groups within the Bacteria and to discern logically their branching order from the common ancestor (Gupta, 1998a, 2000a, b). These signatures also permit the placement of any bacterial species into one of these groups based on the presence or absence of specific signatures in different proteins (Gupta et al., 1999; Gupta, 2000a).

In the present work, the signature sequence approach was used to determine the phylogenetic placement of Fibrobacter succinogenes within the domain Bacteria. Results presented here show that $F$. succinogenes is most closely related to the Chlamydia and CFB groups of species. A summary of the signature sequences that were used to derive this inference is presented in Fig. 6. The presence of the large 21 aa insert in $F$. succinogenes Hsp70, which is distinctive of all Gram-negative bacteria and not found in any Gram-positive bacteria, places Fibrobacter in the Gram-negative group. This indel has previously been shown to be due to an insertion in the common ancestor of Gram-negative bacteria, rather than a deletion in the Gram-positive group. This makes the Gram-positive bacteria an ancestral lineage and it provides a reference point for interpreting other signature sequences. The placement of Fibrobacter in the Gram-negative group is also supported by the signature in the Hsp60 protein, which additionally also excludes Fibrobacter from the Deinococcus-Thermus group and the green non-sulphur bacteria, which are two of the earliest branching divisions among Gram-negative bacteria (Gupta, 1998a). A key signature for the phylogenetic placement of $F$. succinogenes is found in the protein Ala-tRNA synthetase. A 4 aa insert present in this protein is found only in Proteobacteria and in the Chlamydia and CFB groups of species (Gupta, 2000a). Since this insert is also present in the F. succinogenes homologue, it provides strong evidence that F. succinogenes is related to one of these taxa. The presence of this insert also excludes $F$. succinogenes from the earlier branching spirochaetes and the cyanobacterial divisions. The distinction whether F. succinogenes belongs to the proteobacterial group or to the Chlamydia-CFB group is made on the basis of two signature sequences in CTP synthase and Hsp70 proteins that are distinctive of the proteobacterial species. The absence of these signatures in the $F$. succinogenes homologues provides evidence that this species does not belong to the proteobacterial group and places it in a similar position as the Chlamydia-CFB group.

The placement of Fibrobacter in a similar position as the Chlamydia-CFB group, as carried out in this work, is based on a single type species and further confirmation of this inference with additional type species would be 
Fibrobacter succinogenes

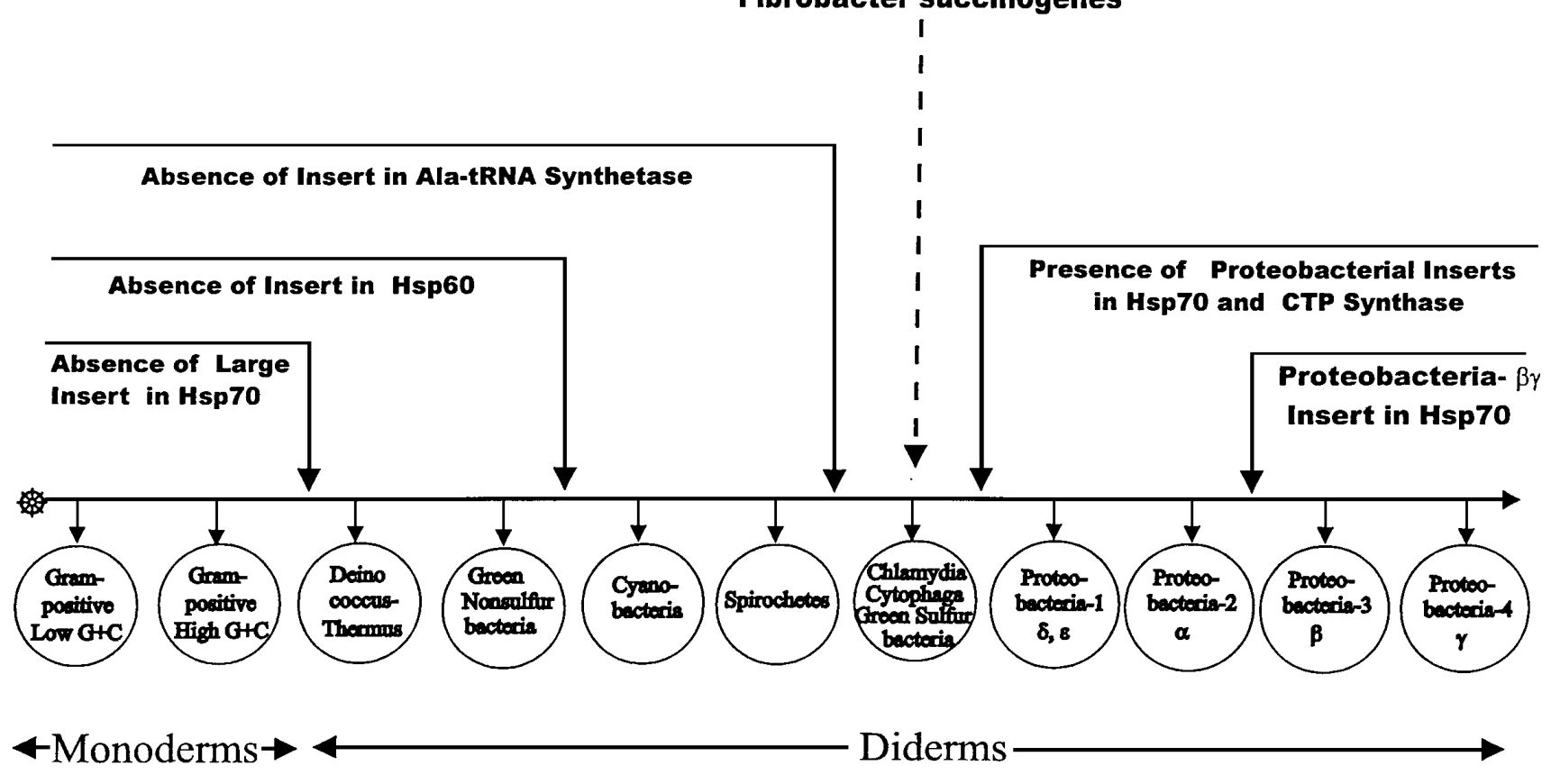

Fig. 6. A summary diagram showing the deductive process used to determine the branching position of species. The arrows indicate the stages where the signature sequences in different proteins examined in the present work were introduced. All groups underneath the arrows share the indicated indels and no exceptions to these were observed. The other signatures used to deduce the branching orders of various groups have been described previously (Gupta, 1998a, 2000a) and are not shown here. Monoderms (cell types bounded by a single membrane) are shown to be ancestral to the diderms (bounded by two different membranes) (Gupta, 1998a, 2000a). Based on different signatures, F. succinogenes is indicated to have evolved after the branching of spirochaetes and other groups preceding it, but before the branching of different divisions of Proteobacteria.

very helpful. However, a specific relationship of $F$. succinogenes to the Chlamydia-CFB group, which includes the Bacteroides family, is supported by the fact that, in a similar manner to $F$. succinogenes, many other members of this group are able to digest and grow on complex substrates such as cellulose, chitin and agar (Hungate, 1950; Paster et al., 1994; Reichenbach, 1992; Shah, 1992; Weisburg et al., 1985; Woese, 1987). F. succinogenes, originally known as Bacteroides succinogenes, was first placed in the Bacteroidaceae family (Holdeman et al., 1984). Subsequent phylogenetic studies based on $16 \mathrm{~S}$ rRNA indicated that $F$. succinogenes was less closely related to other species of Bacteroides, including the type species Bacteroides fragilis as compared to species belonging to other families such as Flavobacterium heparinum (Amann et al., 1992; Montgomery et al., 1988). Although, based on these observations, F. succinogenes was transferred to the new genus Fibrobacter, these studies still indicate that in comparison to the other divisions within the Bacteria, members of the CFB group are the closest relatives of Fibrobacter. The relative branching order of different species within the Chlamydia-CFBFibrobacter group is presently unclear. It is expected that as additional signature sequences that enable further distinction between this group of species are identified, the relationship between members of this diverse group will become clearer.
Lastly, it should be emphasized that the use of signature sequences for the phylogenetic placement of species into different groups, or to understand the relative branching orders of these groups, is not contradictory to the phylogenetic analysis based on $16 \mathrm{~S}$ rRNA, but it complements such analyses in important respects. In our earlier work, where placement of a large number of bacterial species into different groups was carried out using the signature sequence approach, a nearly perfect correlation was observed with the assignment based on the 16S rRNA trees (Gupta, 1998a, 2000a). However, the main advantage of the signature approach lies in the fact that it allows deduction of the branching orders of the main bacterial taxa, which thus far cannot be reliably determined based on the rRNA or other gene/protein phylogenies. The signature sequences in various proteins also permit a more precise molecular definition of different bacterial groups (or means to distinguish between them) than has hitherto been possible based on the phylogenetic trees. In these regards the signature sequence approach complements the major deficiencies of the rRNA trees in clarifying/ understanding the genealogy of the Bacteria. It should however be mentioned that although the signature sequences are able to make clear distinction between different groups, they do not provide detailed information regarding the interrelationship of various species within a given group. Such information is contained in 
the phylogenetic trees. Hence, the use of signature sequences and the analysis based on phylogenetic trees provide complementary, and not contradictory, approaches for understanding the evolutionary relationships among prokaryotes.

\section{ACKNOWLEDGEMENTS}

The work was supported by supported by a research grant from the Medical Research Council (Canadian Institute of Health Research) of Canada. We are grateful to Drs C. Forsberg and J. Yanke for providing us with F. succinogenes DNA.

\section{REFERENCES}

Amann, R. L., Lin, C., Key, R., Montgomery, L. \& Stahl, D. A. (1992). Diversity among Fibrobacter isolates: towards a phylogenetic classification. Syst Appl Microbiol 15, 23-31.

Asanuma, N., Iwamoto, M. \& Hino, T. (1999). Effect of the addition of fumarate on methane production by ruminal microorganisms in vitro. J Dairy Sci 82, 780-787.

Brown, J. R. \& Doolittle, W. F. (1995). Root of the universal tree of life based on ancient aminoacyl-tRNA synthetase gene duplications. Proc Natl Acad Sci US A 92, 2441-2445.

Cheng, K. J., Forsberg, C. W., Minato, H. \& Costerton, J. W. (1991). Microbial ecology and physiology of feed degradation within the rumen. In Physiological Aspects of Digestion and Metabolism in Ruminants, pp. 595-624. Edited by T. Tsuda, Y. Sasaki \& R. Kawahima. New York: Academic Press.

Debroas, D. \& Blanchart, G. (1993). Interactions between proteolytic and cellulolytic rumen bacteria during hydrolysis of plant cell wall protein. Reprod Nutr Dev 33, 283-288.

Doolittle, W. F. (1999). Phylogenetic classification and the universal tree. Science 284, 2124-2128.

Fields, M. W., Mallik, S. \& Russell, J. B. (2000). Fibrobacter succinogenes $\mathrm{S} 85$ ferments ball-milled cellulose as fast as cellobiose until cellulose surface area is limiting. Appl Microbiol Biotechnol 54, 570-574.

Fraser, C. M., Gocayne, J. D., White, O. \& 7 other authors (1995). The minimal gene complement of Mycoplasma genitalium. Science 270, 397-403.

Galley, K. A., Singh, B. \& Gupta, R. S. (1992). Cloning of HSP70 (dnaK) gene from Clostridium perfringens using a general polymerase chain reaction based approach. Biochim Biophys Acta 1130, 203-208.

Garcia-Vallve, S., Romeu, A. \& Palau, J. (2000). Horizontal gene transfer of glycosyl hydrolases of the rumen fungi. Mol Biol Evol $17,352-361$.

Gokarn, R. R., Eitnan, M. A., Martin, S. A. \& Eriksson, K. E. (1997). Production of succinate from glucose, cellobiose, and various cellulosic materials by the ruminal anaerobic bacteria Fibrobacter succinogenes and Ruminococcus flavefaciens. Appl Biochem Biotechnol 68, 69-80.

Gupta, R. S. (1995). Evolution of the chaperonin families (Hsp60, Hsp10 and Tcp-1) of proteins and the origin of eukaryotic cells. Mol Microbiol 15, 1-11.

Gupta, R. S. (1998a). Protein phylogenies and signature sequences: a reappraisal of evolutionary relationships among Archaebacteria, Eubacteria, and Eukaryotes. Microbiol Mol Biol Rev 62, 1435-1491.

Gupta, R. S. (1998b). What are archaebacteria: life's third domain or monoderm prokaryotes related to Gram-positive bacteria? A new proposal for the classification of prokaryotic organisms. Mol Microbiol 29, 695-708.

Gupta, R. S. (2000a). The phylogeny of Proteobacteria: relationships to other Eubacterial phyla and eukaryotes. FEMS Microbiology Reviews 24 , 367-402.

Gupta, R. S. (2000b). The natural relationships among prokaryotes. CRC Crit Rev Microbiol 26, 111-131.

Gupta, R. S. (2000c). Evolutionary relationships among bacteria: does 16S rRNA provide all the answers? ASM News 66, 189-190.

Gupta, R. S. \& Golding, G. B. (1993). Evolution of HSP70 gene and its implications regarding relationships between archaebacteria, eubacteria, and eukaryotes. J Mol Evol 37, 573-582.

Gupta, R. S. \& Singh, B. (1992). Cloning of the HSP70 gene from Halobacterium marismortui: relatedness of archaebacterial HSP70 to its eubacterial homologs and a model for the evolution of the HSP70 gene. J Bacteriol 17, 4594-4605.

Gupta, R. S., Mukhtar, T. \& Singh, B. (1999). Evolutionary relationships among photosynthetic prokaryotes (Heliobacterium chlorum, Chloroflexus aurantiacus, Cyanobacteria, Chlorobium tepidum and Proteobacteria): implications regarding the origin of photosynthesis. Mol Microbiol 32, 893-906.

Holdeman, L. V., Kelley, R. W. \& Moore, W. E. C. (1984). Family I. Bacteroidaceae Pribam 1933. In Bergey's Manual of Systematic Bacteriology, pp. 602-662. Edited by N. R. Krieg \& J. G. Holt. Baltimore: Williams \& Wilkins.

Huang, L. \& Forsberg, C. W. (1990). Cellulose digestion and cellulase regulation and distribution in Fibrobacter succinogenes subsp. succinogenes S85. Appl Environ Microbiol 56, 1221-1228.

Hugenholtz, P., Pitulle, C., Hershberger, K. L. \& Pace, N. (1998). Novel division level bacterial diversity in a Yellowstone hot spring. J Bacteriol 180, 366-376.

Hungate, R. E. (1950). The anaerobic mesophilic cellulolytic bacteria. Bacteriol Rev 14, 1-49.

Iwabe, N., Kuma, K., Hasegawa, M., Osawa, S. \& Miyata, T. (1989). Evolutionary relationship of archaebacteria, eubacteria, and eukaryotes inferred from phylogenetic trees of duplicated genes. Proc Natl Acad Sci US A 86, 9355-9359.

Lindquist, S. \& Craig, E. A. (1988). The heat-shock proteins. Annu Rev Genet 22, 631-677.

Ludwig, W. \& Schleifer, K. H. (1999). Phylogeny of Bacteria beyond the 16S rRNA Standard. ASM News 65, 752-757.

Maidak, B. L., Cole, J. R., Parker, C. T., Jr and 11 other authors (1999). A new version of the RDP (Ribosomal Database Project). Nucleic Acids Res 27, 171-173.

Montgomery, L., Flesher, B. \& Stahl, D. (1988). Transfer of Bacteroides succinogenes (Hungate) to Fibrobacter gen. nov. as Fibrobacter succinogenes comb. nov. and description of Fibrobacter intestinalis sp. nov. Int J Syst Bacteriol 38, 430-435.

Olsen, G. J. \& Woese, C. R. (1993). Ribosomal RNA: a key to phylogeny. FASEB J 7, 113-123.

Paster, B. J., Dewhirst, F. E., Olsen, I. \& Fraser, G. J. (1994). Phylogeny of Bacteroides, Prevotella, and Porphyromonas spp. and related bacteria. J Bacteriol 176, 725-732.

Reichenbach, H. (1992). The Order Cytophagales. In The Prokaryotes, pp. 3631-3675. Edited by A. Balows, H. G. Trüper, M. Dworkin, W. Harder \& K. H. Schleifer. New York: Springer.

Rusanganwa, E., Singh, B. \& Gupta, R. S. (1992). Cloning of HSP60 (GroEL) operon from Clostridium perfringens using a polymerase chain reaction based approach. Biochim Biophys Acta 1130, 90-94. 
Shah, R. N. (1992). The genus Bacteroides and related taxa. In The Prokaryotes, pp. 3593-3607. Edited by A. Balows, H. G. Trüper, M. Dworkin, W. Harder \& K. H. Schleifer. New York: Springer.

Shi, Y., Odt, C. L. \& Weimer, P. J. (1997). Competition for cellulose among three predominant ruminal cellulolytic bacteria under substrate-excess and substrate-limited conditions. Appl Environ Microbiol 63, 734-742.

Wang, Y., McAllister, T. A., Yanke, L. J. \& Cheeke, P. R. (2000). Effect of steroidal saponin from Yucca schidigera extract on ruminal microbes. J Appl Microbiol 88, 887-896.

Weimer, P. J., Waghorn, G. C., Odt, C. L. \& Mertens, D. R. (1999). Effect of diet on populations of three species of ruminal cellulolytic bacteria in lactating dairy cows. J Dairy Sci 82, 122-134.
Weisburg, W. G., Oyaizu, Y., Oyaizu, H. \& Woese, C. R. (1985). Natural relationship between Bacteroides and Flavobacteria. J Bacteriol 164, 230-236.

Woese, C. R. (1987). Bacterial evolution. Microbiol Rev 51, 221-271.

Woese, C. R. (1998). The universal ancestor. Proc Natl Acad Sci U S A 95, 6854-6859.

Zinder, S. H. (1998). Bacterial diversity. In Topley and Wilson's Microbiology and Microbial Infections, 9th edn, vol. 2, pp. 125-147. Edited by A. Balows \& B. I. Duerden. London: Arnold.

Received 15 February 2001; revised 17 May 2001; accepted 25 May 2001. 